\title{
Recurrent Sebaceous Gland Carcinoma of Upper Eyelid - A Case Report
}

\author{
${ }^{1}$ Sheeba Bhardwaj (Junior resident, Radiation Oncology) \\ ${ }^{2}$ Peoli Mukutawat (Junior resident, Radiation Oncology) \\ ${ }^{3}$ Vivek Kaushal (Senior Professor and Head, Radiation Oncology) \\ Senior girl's hostel Pandit B.D Sharma PGIMS, Rohtak
}

\begin{abstract}
Sebaceous gland carcinoma of ocular adnexa is a highly malignant but relatively rare condition. It has an aggressive course with tendency for local invasion and systemic metastasis resulting in poor outcome and increased mortality. Here, we describe the clinical course of recurrent sebaceous gland carcinoma of the upper eyelid in a 55 year old female patient who presented with complaint of growth over right upper eyelid from 10 years.
\end{abstract}

\section{INTRODUCTION}

Sebaceous gland carcinoma of ocular adnexa is a highly malignant but relatively rare condition. It is considered as the third most common eyelid malignancy after basal and squamous cell carcinomas. Most commonly it is seen in elder population with female predisposition. The reported incidence is less than $1 \%$ of all skin malignancies. ${ }^{1}$ It accounts for $1-5.5 \%$ of all eyelid malignancies with more predilections for upper eyelid. ${ }^{2}$ It may originate from the meibomian glands in the tarsus, zeis glands at the eyelid margin or sebaceous glands at caruncle or eyebrows. It can also spread through the canaliculus to the lacrimal system and to nasal cavity. ${ }^{3}$ The tumor is also called as adenocarcinoma of sebaceous gland, meibomian gland carcinoma, or Zeis gland carcinoma. 5\% cases may have simultaneous involvement of both the eyelids. ${ }^{4}$ It has great resemblance to other benign or malignant lesions and the differential diagnosis includes chalazion, blepharitis, conjunctivitis, basal cell carcinoma, squamous cell carcinoma, leukoplakia, ocular pemphigoid and carcinoma in-situ..$^{5}$ Diagnosis and therapy tend to be delayed because sebaceous carcinoma is often mistaken for benign entities such as chalazion, conjunctivitis, or blepharitis. ${ }^{6}$

\section{CASE REPORT}

A 55 year old female presented with complaint of growth over right upper eyelid (figure-1) from 10 years. Initially she noticed a small nodule over eyelid which was progressive in nature. Patient underwent resection of the nodule. Histopathologic examination showed sebaceous gland carcinoma. After 1 year of resection she again developed growth over right upper eyelid for which she received 6- cycles of chemotherapy with TPF regimen (taxanes, platins, 5-FU). After i.v. chemotherapy patient received metronomic chemotherapy with cyclophosphamide following which there was a reduction in the size of the growth. Patient remained asymptomatic for a period of 6 months after which she again developed growth over right upper eyelid which was rapidly progressive with off and on bleeding and later the lesion was infested by maggots. MRI showed an ill-defined lesion measuring $79 \mathrm{~mm} \times 41 \mathrm{~mm} \times 34 \mathrm{~mm}$ in right peri-orbital region involving the right eyelid with involvement of extra ocular muscles and right lacrimal gland. Lesion also involved the infratemporal fossa and ipsilateral nasolacrimal duct. For these complaints patient underwent orbital exenteration (figure-2). Few days after exenteration patient again developed swelling in pre-auricular region. Patient is currently on tab cyclophosphamide and is on regular follow up.

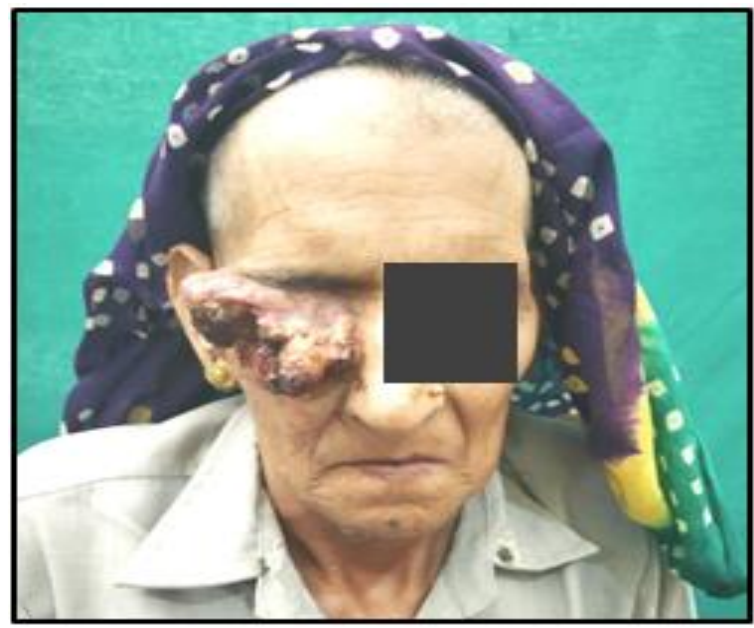

Fig 1:- Growth arising from right upper eyelid at presentation

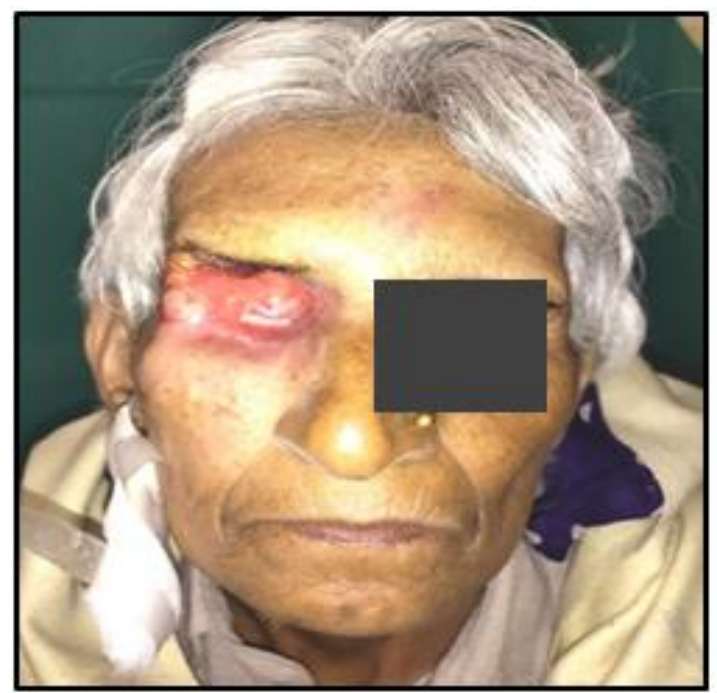

Fig 2:- Orbital exenteration 


\section{DISCUSSION}

Sebaceous gland carcinoma of ocular adnexa is a highly malignant but relatively rare condition and it accounts for $1-5.5 \%$ of all eyelid malignancies with more predilections for upper eyelid. The primary treatment of sebaceous gland carcinoma surgery which includes local excision or orbital exenteration in advanced cases. Other treatment modalities are mitomycin $\mathrm{C}$, cryotherapy, and radiotherapy. $^{7}$ Involvement of regional lymph nodes requires neck dissection. Adjuvant chemotherapy and radiotherapy are required if distant metastasis is present. The tumor is frequently mistaken for blepharoconjunctivitis or a chalazion which results in delayed diagnosis and poor prognosis. ${ }^{8}$ The mortality rate is $5-10 \%$ because of delayed diagnosis and treatment. The distant metastasis has $25 \%$ mortality rate. ${ }^{9}$ The case described in this report had a long history of nodule over right upper eyelid for which she underwent resection. After resection she again developed growth over right upper eyelid for which she received 6cycles of chemotherapy with TPF regimen followed by oral chemotherapy with cyclophosphamide. The chemotherapy temporarily controlled the growth but it started to grow aggressively for which orbital exenteration was done. The course of disease in this patient confirms the aggressive nature of the disease with very high rate of recurrence.

\section{CONCLUSION}

Sebaceous gland carcinomas have a high incidence of recurrence; therefore, continuous surveillance is necessary for early detection of recurrence or secondaries from the lesion. Also it has aggressive behavior which results in early metastasis and is associated with high mortality rate. Early diagnosis and treatment may decrease the long-term morbidity and extend the survival rate of such patients.

\section{REFERENCES}

[1]. Shurti Singh, Swarn Kaur, Alok Mohan, Sunder Goyal. Sebaceous carcinoma of right upper eyelid: case report and literature review. International Journal of Cancer Therapy and Oncology. 2013;1(3):1-3.

[2]. Gardetto A, Rainer C, Ensinger C, Baldissera I, PizaKatzer H. Sebaceous carcinoma of the eyelid: A rarity worth considering. Br J Ophthalmol.2002;86:243-4.

[3]. Khan JA, Grove AS Jr, Joseph MP, Goodman M. Sebaceous carcinoma. Diuretic use, lacrimal system spread, and surgical margins. Ophthal Plast Reconstr Surg 1989;5:227-34.

[4]. Achyut Narain Pandey, Amit Raina, Deepa Hatwal, et al. A Rare Case of Sebaceous Cell Carcinoma of Lower Lid. Ophthalmol Res Rep. 2017;1(1):1-3.

[5]. Shields JA, Demirci H, Marr BP, Eagle RC Jr, Shields CL. Sebaceous carcinoma of the eyelids: personal experience with 60 cases. Ophthalmology. 2004;111(12):2151-7.

[6]. Meenu Gill, Shilpa Garg, Rajnish Kalra, Rajeev Sen Sebaceous carcinoma of the eyelid diagnosed on fine needle aspiration cytology. J Cytol.2012;29(1):75-6.
[7]. Muqit MM, Roberts F, Lee WR, Kemp E. Improved survival rates in sebaceous carcinoma of the eyelid. Eye (Lond) 2004;18:49-53.

[8]. Condon GP, Brownstein S, Codère F. Sebaceouscarcinoma of the eyelid masquerading as superiorlimbic keratoconjunctivits. Arch Ophthalmol 1985;103:1525-9.

[9]. Wali UK, Al-Mujaini A. Sebaceous gland carcinoma of the eyelid. Oman J Ophthalmol 2010;3:117-21. 